\title{
The production of HCN dimer and more complex oligomers in dense interstellar clouds
}

\author{
I. W. M. Smith ${ }^{1}$, D. Talbi ${ }^{2}$, and E. Herbst ${ }^{3}$ \\ 1 School of Chemistry, The University of Birmingham, Edgbaston, Birmingham B15 2TT, UK \\ 2 LETMEX, Université de Nice Sophia Antipolis, Parc Valois, U.F.R. Sciences, 06108 Nice Cedex 2, France \\ 3 Departments of Physics and Astronomy, The Ohio State University, Columbus, OH 43210, USA
}

Received 8 January 2001 / Accepted 16 January 2001

\begin{abstract}
Using quantum chemical and kinetic techniques, we show that the reaction between two HCN molecules under interstellar conditions cannot lead efficiently to a dimer of this species $\left(\mathrm{H}_{2} \mathrm{C}_{2} \mathrm{~N}_{2}\right)$, whatever its structure. If the dimerization reaction does not occur efficiently, then it is impossible to synthesize detectable abundances of the pre-biotic $\mathrm{HCN}$ oligomer adenine $\left(\mathrm{H}_{5} \mathrm{C}_{5} \mathrm{~N}_{5}\right)$ in the interstellar medium via successive neutral-neutral reactions between $\mathrm{HCN}$ and its dimer, trimer, and tetramer.
\end{abstract}

Key words. ISM: abundances - ISM: molecules

\section{Introduction}

Mechanisms for the formation of pre-biotic molecules have been discussed in the scientific literature for at least four decades. The "oligomerization" of HCN provides a particularly intriguing route to chemical evolution because it may lead to the production of biologically important molecules such as adenine. Adenine has the molecular formula $\mathrm{C}_{5} \mathrm{H}_{5} \mathrm{~N}_{5}$ and can therefore be viewed as a pentamer of $\mathrm{HCN}$, a molecule that is quite abundant under primitive conditions in the universe and the early Earth. Until recently, speculation about the role of $\mathrm{HCN}$ in forming prebiotic molecules had been confined to reactions in solution under conditions that might be expected to have arisen on the early Earth (Oró 1961). Chakrabarti \& Chakrabarti (2000a) on the basis of modelling calculations have now boldly proposed, however, that DNA bases can be produced by gas-phase reactions in dense interstellar clouds, both in quiescent cores and in star-forming regions.

They postulated that adenine could be formed in the four successive steps:

$\mathrm{HCN}+\mathrm{HCN} \longrightarrow \mathrm{CH}(\mathrm{NH}) \mathrm{CN}$,

$\mathrm{CH}(\mathrm{NH}) \mathrm{CN}+\mathrm{HCN} \longrightarrow \mathrm{NH}_{2} \mathrm{CH}(\mathrm{CN})_{2}$,

$\mathrm{NH}_{2} \mathrm{CH}(\mathrm{CN})_{2}+\mathrm{HCN} \longrightarrow \mathrm{NH}_{2}(\mathrm{CN}) \mathrm{C}=\mathrm{C}(\mathrm{CN}) \mathrm{NH}_{2}$,

$\mathrm{NH}_{2}(\mathrm{CN}) \mathrm{C}=\mathrm{C}(\mathrm{CN}) \mathrm{NH}_{2}+\mathrm{HCN} \longrightarrow \mathrm{C}_{5} \mathrm{H}_{5} \mathrm{~N}_{5}$ (adenine).

Send offprint requests to: E. Herbst, e-mail: herbst@mps.ohio-state.edu
For each of the steps in this condensation mechanism, they assumed a temperature-independent rate coefficient of $k=10^{-10} \mathrm{~cm}^{3} \mathrm{~s}^{-1}$, a value close to the collisionallydetermined limit for collisions between two electrically neutral species. With this input, on modelling molecular evolution in both a collapsing and a quiescent interstellar cloud core, they found significant, indeed considerable, formation of adenine on relatively short time-scales $\left(10^{6} \mathrm{yr}\right)$. The purpose of the present paper is to look at one of the assumptions of Chakrabarti \& Chakrabarti (2000a) in detail. In particular, we bring quantum chemical and kinetic arguments to bear on the first step of the proposed synthesis of adenine: the dimerization of $\mathrm{HCN}$. We find that the reaction cannot occur efficiently under typical lowtemperature (10-100 K) interstellar conditions or even at considerably higher temperatures.

\section{Quantum chemical calculations}

An extensive search for a pathway connecting two isolated $\mathrm{HCN}$ molecules to an $\mathrm{H}_{2} \mathrm{C}_{2} \mathrm{~N}_{2}$ chemically-bound complex was undertaken using the density functional formalism. The B3LYP functional (Becke 1993) available in the Gaussian 98 package (Frisch et al. 1998) was used. The surface mapping was determined using a $6-31 \mathrm{G}^{* *}$ basis set and the critical points (minima and transition states) were re-optimized with the more extensive $6-311+\mathrm{G}(2 \mathrm{df}, \mathrm{pd})$ basis set. Unless indicated, for more accurate electronic energies, single point calculations were performed at the MP4SDTQ/6-311+G(2df,pd) level of theory. This is a fourth order Moller-Plesset perturbative 
Table 1. MP4SDTQ/6-311+G(2df,pd) relative energies

\begin{tabular}{lr}
\hline Species & Relative Energy $(\mathrm{kcal} / \mathrm{mol})$ \\
\hline HCN + HCN & 0.0 \\
Isomer I & -3.99 \\
Isomer II & -3.68 \\
Isomer III & -3.10 \\
Isomer IV & +3.10 \\
Isomer V & +12.5 \\
Transition State & +71 \\
\hline
\end{tabular}

Notes: The energies include B3LYP/6-311+G(2df,pd) zeropoint energy corrections scaled by 0.9613 . For isomer $\mathrm{V}$ and for the transition state, the energies were calculated at the MP4SDTQ/6-311+G(df,pd) level. B3LYP/6-311+G(2df,pd) otimzed geometries were used in all cases.

calculation including contributions from single, double, triple and quadruple excitations. All energies have been corrected for zero-point energy (ZPE) contributions calculated at the B3LYP / 6-311+G(2df,pd) level and scaled by a factor of 0.9613 (Frisch et al. 1996). In a previous work, Clemmons et al. (1983) characterized all possible isomers of the $\mathrm{H}_{2} \mathrm{C}_{2} \mathrm{~N}_{2}$ formula and predicted that three chemically-bound isomers are lower in energy than two non-interactive HCN monomers.

In Fig. 1 we show our B3LYP/6-311+G(2df,pd) optimized geometries for the five lowest $\mathrm{H}_{2} \mathrm{C}_{2} \mathrm{~N}_{2}$ isomers, including the linear $(\mathrm{HCN})_{2}$ hydrogen-bonded van der Waals dimer. Corresponding MP4SDTQ relative energies for which ZPE corrections have been considered are reported in Table 1. Our calculations show that only two chemically-bound isomers (structures II \& III) as well as the linear van der Waals dimer (structure I) are lower in energy than two HCN monomers, while the chemicallybound isomers IV \& V are higher in energy. Most importantly, none of the dimer structures is strongly bound with respect to the individual HCN units. Structure II, which possesses the lowest energy of the chemically-bound dimers, and which has the structure of the product of reaction $(1)$, lies only $3.68 \mathrm{kcal} / \mathrm{mol}(1 \mathrm{kcal} / \mathrm{mol}=503 \mathrm{~K})$ below HCN + HCN. This is not very different from the weak binding energy of the van der Waals dimer (3.99 kcal $/ \mathrm{mol})$. The calculated value of this binding energy is close to the calorimetric one of $3.28 \mathrm{kcal} / \mathrm{mol}$ (Giauque et al. 1939) and the spectroscopic one (with our calculated zero-point energy correction) of $3.58 \mathrm{kcal} / \mathrm{mol}$ (Buxton et al. 1981).

In a search at the B3LYP/6-31G** level for a pathway connecting the two separated $\mathrm{HCN}$ species to isomers II \& III, two reaction coordinates were considered: the distance between carbon atoms on the respective $\mathrm{HCN}$ monomers for the case of either a parallel or an antiparallel approach, and the distance between the $\mathrm{N}$ atom of one monomer and the $\mathrm{C}$ atom of the other monomer for the case of a perpendicular approach. In all cases, all other parameters (distances and angles) were allowed to relax for each $\mathrm{C}-\mathrm{C}$ or $\mathrm{C}-\mathrm{N}$ distance. None of these approaches revealed any low energy path to the formation of a chemically-bound $\mathrm{H}_{2} \mathrm{C}_{2} \mathrm{~N}_{2}$ isomer. All energy paths lead either to the van der Waals dimer structure I or to the separated HCN species.

Having failed to locate any low energy (barrierless) path from two separate $\mathrm{HCN}$ molecules to a chemicallybound $\mathrm{H}_{2} \mathrm{C}_{2} \mathrm{~N}_{2}$ complex, we tried to locate a low transition state with a structure intermediate between the two monomers and the low energy chemically-bound $\mathrm{H}_{2} \mathrm{C}_{2} \mathrm{~N}_{2}$ isomers. The lowest transition state we found at the B3LYP/6-31G** level and confirmed at the B3LYP/6$311+\mathrm{G}(2 \mathrm{df}, \mathrm{pd})$ level (see Fig. 1) has, taking into account the scaled ZPE corrections, an energy $71 \mathrm{kcal} / \mathrm{mol}$ above the separated HCN species, at the MP4SDTQ/6$311+\mathrm{G}(\mathrm{df}, \mathrm{pd})$ level. The B3LYP/6-311+G(2df,pd) vibrational analysis of this transition state shows one imaginary frequency of $886 i \mathrm{~cm}^{-1}$. In order to search for the minima associated with this transition state, the geometry of the transition state was slightly distorted and the geometry optimization routine used to locate the appropriate minima, through the steepest descent method (Fletcher \& Powell 1963). Stretching the C-C bond by $0.05 \AA$ leads to the hydrogen-bonded dimer (isomer I) and subsequently to the two monomers. Shortening the same C-C bond by $0.05 \AA$ leads analogously to the high energy, chemically-bound, $\mathrm{H}_{2} \mathrm{C}_{2} \mathrm{~N}_{2}$ complex, isomer V. All other combinations of distortions from the transition state also lead to dissociation into $\mathrm{HCN}+\mathrm{HCN}$ via the van der Waals dimer or to formation of the high energy isomer V. Implementation of these procedures demonstrates that the identified transition state is associated with the van der Waals dimer and the isomer $\mathrm{V}$, as is shown schematically in Fig. 2. From these quantum chemical investigations, we suggest that there are no low energy paths to any of the chemically-bound dimers II-IV.

\section{Rate coefficients for dimer formation}

Independently of what particular chemically-bound isomer of the dimer of $\mathrm{HCN}$ is produced, the activation energy found $-71 \mathrm{kcal} / \mathrm{mol}(36000 \mathrm{~K})$ - is far higher than $k_{\mathrm{B}} T$ in even warm interstellar regions surrounding newborn stars. The rate coefficient $k_{\mathrm{dim}}\left(\mathrm{cm}^{3} \mathrm{~s}^{-1}\right)$ for formation of such an isomer is given by the expression

$k_{\operatorname{dim}}(T) \sim 110^{-10} \exp (-36000 / T)$

if we assume a relatively favorable value for the so-called "pre-exponential factor" (Smith 1980). In the models of Chakrabarti \& Chakrabarti (2000a), the temperature is $10 \mathrm{~K}$ throughout their static cloud core and at the same temperature for much of the collapsing core before starting to rise at a distance of $10^{15} \mathrm{~cm}(67 \mathrm{AU})$ from the proto-star and eventually reaching $1000 \mathrm{~K}$ at a distance $1 \mathrm{AU}$ from this object. Even at a temperature of $1000 \mathrm{~K}$, the estimated rate coefficient for HCN dimerization is many orders of magnitude less than the assumed value of $10^{-10} \mathrm{~cm}^{3} \mathrm{~s}^{-1}$. It is likely, but not proven, that the 


$$
\underset{1.068}{\mathrm{H}-\mathrm{C}} \underset{1.144}{\mathrm{I}} \underset{2.224}{\mathrm{~N}-\mathrm{-}} \mathrm{-H} \underset{1.074}{-} \mathrm{C} \underset{1.147}{-} \mathrm{N}
$$

II

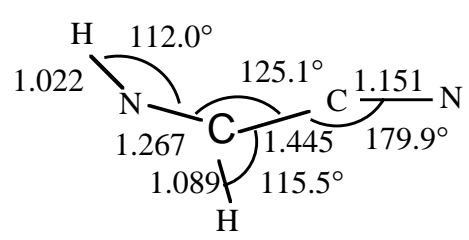

IV

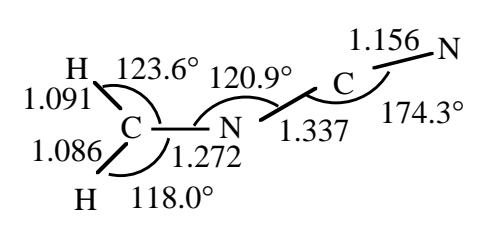

TS

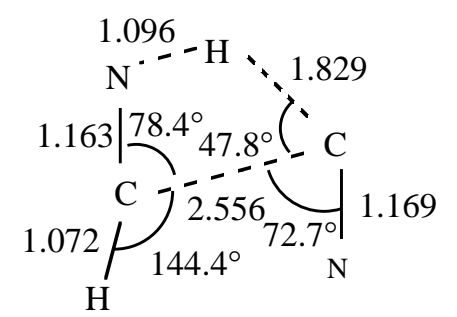

III

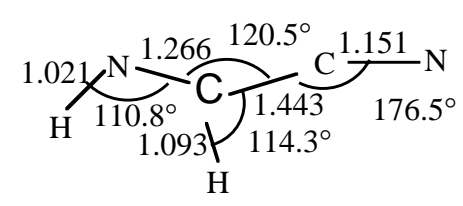

$\mathbf{V}$

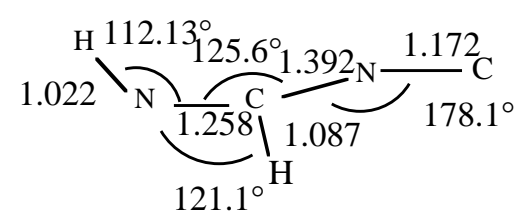

Fig. 1. Structures of the five lowest-energy HCN dimer isomers including the van der Waals dimer (I) as well as the transition state between structures I and V optimized at the B3LYP/6-311+G(2df,pd) level. The distances are in $\AA$ and the angles in degrees

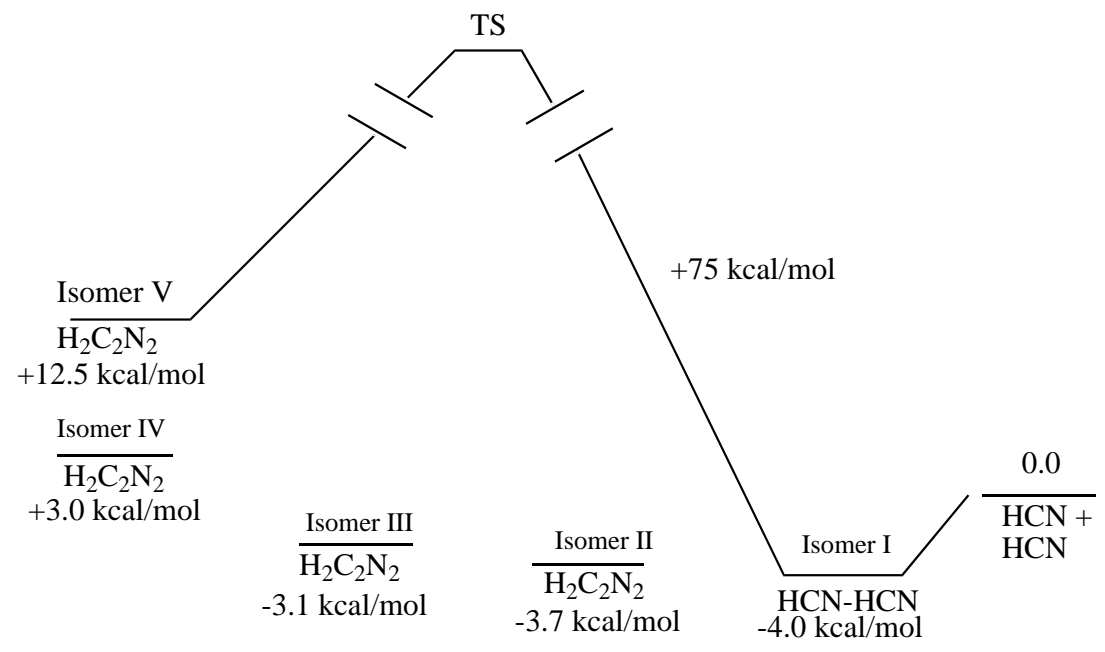

Fig. 2. The lowest energy reaction pathway for formation of a chemically-bound HCN dimer. ZPE corrections are included. The calculation was performed at the MP4SDTQ/6-311+G(2df,pd) level for isomers I, II, III, IV, and at the MP4SDTQ/6$311+\mathrm{G}(\mathrm{df}, \mathrm{pd})$ for isomer $\mathrm{V}$ and for the transition state. In all cases B3LYP/6-311+G(2df,pd) optimized geometries were used 
other rate coefficients in the oligomerization of $\mathrm{HCN}$ are also very small, since large activation energies are typically expected for reactions between neutral species that are non-radicals.

A second aspect of reaction (1), and of all the other reactions in the reaction sequence (1)-(4), makes it even less likely that the rate coefficients approach the collision limit under interstellar conditions. This aspect is that they are association reactions; that is, reactions in which two species combine to form one molecule. Such reactions require a mechanism for the loss of energy from the energized complex which is initially formed (Smith 1980). At the low densities in interstellar clouds, the only mechanism available is spontaneous emission. Radiative association is known to be inefficient for small species unless their enthalpy of formation is large because their lifetime with respect to re-dissociation is much shorter than the radiative lifetime (Smith 1980; Bates \& Herbst 1988). Thus, it is unlikely that the dimerization rate coefficient is even as large as given in Eq. (5).

There is no activation energy associated with the formation of the weakly bound van der Waals dimer (structure I), and so the rate coefficient for this process must be considered separately. For chemically-bound species, rate coefficients for radiative association can be estimated by standard and successful techniques (Bates \& Herbst 1988). These methods require a statistical calculation of the equilibrium between the energized complexes formed in collisions between monomers and the separated monomers themselves, plus an estimate of the first-order rate coefficient $\left(k_{\mathrm{rad}}\right)$ for spontaneous emission from the energized complexes. The values of $k_{\text {rad }}$ are largest $(\sim 100-$ $1000 \mathrm{~s}^{-1}$ ) when the complex has IR-allowed high frequency transitions (e.g. vibrations associated with stretching $\mathrm{C}-\mathrm{H}$ bonds). For the case of a weakly bound dimer, like $(\mathrm{HCN})_{2}$, the limited energy released when the van der Waals bond forms can only be spread among a limited number of oscillators, mainly the low frequency modes associated with formation of the dimer. These will surely have very low Einstein coefficients for spontaneous emission. So, dimerization to form the van der Waals dimer will be especially inefficient. With an assumed value of $10 \mathrm{~s}^{-1}$ for $k_{\mathrm{rad}}$, the "thermal" approach of Herbst (Bates \& Herbst 1988) yields a value of $810^{-18} \mathrm{~cm}^{3} \mathrm{~s}^{-1}$ for $k_{\operatorname{dim}}$ at $10 \mathrm{~K}$ with a calculated temperature dependence of $T^{-2.5}$.

There is another compelling piece of evidence that rate coefficients of the sequence (1)-(4) for temperatures up to room temperature are small. Hydrogen cyanide can easily be prepared in the gas-phase and then stored in clean glass bulbs at pressures of some tens of Torr for several weeks, without noticeable deterioration (Smith 1981). If the rate coefficients were large, $\mathrm{HCN}$ would disappear and be converted to adenine in less than a second under these conditions because adenine lies much lower in energy than do five monomers so that back reaction is inefficient.

\section{Abundance in interstellar clouds}

The total abundance of all forms of the HCN dimer under steady-state interstellar cloud conditions in a static cloud can be estimated with the assumption that the dimer is depleted primarily by reaction with molecular and atomic positive ions (Lee et al. 1996). If we let $f$ represent the fractional abundance with respect to gas density, the steady-state fractional abundance of HCN dimers, is given by the formula

$f_{\text {dimer }} \sim \frac{k_{\mathrm{dim}} f_{\mathrm{HCN}}^{2}}{k_{\mathrm{ion}-\mathrm{mol}} f_{\mathrm{I}^{+}}}$,

where $\mathrm{I}^{+}$stands for the population of reactive ions and $k_{\text {ion-mol }}$ is a generic Langevin ion-molecule reaction rate coefficient $\left(10^{-9} \mathrm{~cm}^{3} \mathrm{~s}^{-1}\right)$. With a typical fractional abundance for reactive ions of $10^{-8}$ and for $\mathrm{HCN}$ of $10^{-8}$ in a core of density $10^{4-5} \mathrm{~cm}^{-3}$ (Lee et al. 1996) we calculate that

$f_{\text {dimer }} \sim k_{\text {dim }} \times 10$.

With the value for $k_{\text {dim }}$ assumed by Charkrabarti \& Chakrabarti (2000a), the dimer of HCN would achieve a steady-state fractional abundance of $10^{-9}$, or $1 / 10$ of the $\mathrm{HCN}$ abundance. Extrapolation of this ratio leads to fractional abundances of $10^{-10}$ for the trimer, $10^{-11}$ for the tetramer, and $10^{-12}$ for adenine at steady state, which is close to the value reported by Charkrabarti \& Chakrabarti (2000a) at $10^{6} \mathrm{yr}$, a time only slightly before steady-state conditions set in for a cloud of density $10^{5} \mathrm{~cm}^{-3}$. However, with our estimated values for $k_{\mathrm{dim}}$, we find

$f_{\mathrm{H}_{2} \mathrm{C}_{2} \mathrm{~N}_{2}}=110^{-9} \exp (-36000 / T)$

for the case of the chemically-bound dimer and

$f_{(\mathrm{HCN})_{2}}=810^{-17}(T / 10)^{-2.5}$

for the van der Waals dimer using the thermal value of the rate coefficient. In either case, the abundance of the dimer is miniscule at all temperatures up to $1000 \mathrm{~K}$. The abundances of the trimer, tetramer, and pentamer will be even smaller! The van der Waals dimer has been searched for unsuccessfully in a variety of interstellar clouds although the upper limits for its abundance are still rather high (Schwenewerk et al. 1985).

\section{Conclusions}

On the basis of the evidence marshalled in the previous paragraphs, our conclusion is that the reaction between two HCN monomers cannot lead to a significant amount of dimer under the conditions prevalent in quiescent or collapsing interstellar cloud cores. The conclusion holds whether the dimer is chemically-bound or a simple van der Waals isomer. For the former, the problem is twofold: there is a large activation energy barrier separating the reactants from the product and, in addition, the reaction is an example of radiative association, which is likely 
to be even less efficient than a standard reaction with activation energy. For the van der Waals isomer, there is no activation energy but the radiative association rate is still very low. In a very recent publication, Chakrabarti \& Chakrabarti (2000b) reduced their earlier rate coefficient for reaction (1) by various amounts to allow for the possibility of radiative association without an activation energy barrier. But the point remains that activation energy barriers are to be expected for radiative association reactions between stable neutral species unless weakly-bound van der Waals isomers are formed. Although the rate coefficients for association reactions to form van der Waals isomers are likely to get larger as the sizes of the products increase (Chakrabarti \& Chakrabarti 2000b), it is clear that such a sequence of reactions has little to do with adenine, which is a strongly chemically-bound species.

If HCN dimerization to form a chemically-bound isomer is as inefficient as we calculate it to be, further synthesis leading to the formation of adenine (a pentamer of $\mathrm{HCN}$ ) via reactions between $\mathrm{HCN}$ and oligomers of this species does not occur. There may be syntheses leading to adenine and other pre-biotic molecules that involve ion-molecule or radical-molecule reactions. It is unlikely, however, that neutral-neutral reactions between stable non-radicals will contribute much to the low-temperature chemistry of interstellar clouds.

Acknowledgements. The Astrochemistry Program at The Ohio State University is supported by The National Science Foundation. Parts of the calculations reported here were supported by the CINES (France); the support is gratefully acknowledged.

\section{References}

Bates, D. R., \& Herbst, E. 1988, in Rate Coefficients in Astrochemistry, ed. T. J. Millar, \& D. A. Williams (Dordrecht: Kluwer), 17

Becke, A. D. 1993, J. Chem. Phys., 98, 5648

Buxton, L. W., Campbell, E. J., \& Flygare, W. H. 1981, Chem. Phys., 56, 399

Chakrabarti, S., \& Chakrabarti, S. K. 2000a, A\&A, 354, L6

Chakrabarti, S., \& Chakrabarti, S. K. 2000b, Ind. J. Phys., $74 \mathrm{~B}, 97$

Clemmons, J. H., Jasien, P. G., \& Dykstra, C. E. 1983, Molec. Phys., 48, 631

Fletcher, R., \& Powell, M. J. D. 1963, Comput. J., 6, 163

Frisch, M. J., Trucks, G. W., \& Cheeseman, J. R. 1996, in Recent Developments and Applications of Modern Density Functional Theory, Theoretical and Computational Chemistry, vol. 4, ed. J. Seminario (Amsterdam: Elsevier), 679

Frisch, M. J., Trucks, G. W., Schlegel, H. B. et al. 1998, Gaussian 1998 (Revision A.5), (Pittsburgh: Gaussian, Inc.)

Giauque, W. F., \& Ruehrwein, R. A. 1939, J. Am. Chem. Soc., 61,2626

Lee, H.-H., Bettens, R. P. A., \& Herbst, E. 1996, A\&AS, 119, 111

Oró, J. 1961, Nature, 1961, 1193

Schwenewerk, M. S., Jewell, P. R., Snyder, L. E. et al. 1985, ApJ, 296, 218

Smith, I. W. M. 1980, Kinetics and Dynamics of Elementary Gas Rections (London: Butterworths)

Smith, I. W. M. 1981, J. Chem. Soc. Faraday Trans. 2, 77, 2357 